\title{
Investigation of hydrodynamic and heat transfer characteristics of gas-liquid Taylor flow in vertical capillaries
}

\author{
Jingzhi Zhang ${ }^{1,2}$, Wei Li ${ }^{*}$
}

1. Department of Energy Engineering, Zhejiang University, Hangzhou 310027, China

2. Department of Energy Engineering, Co-Innovation Center for Advanced Aero-Engine, Zhejiang University, Hangzhou 310027, China;

* Corresponding author. Tel./fax: +86 57187952244.

E-mail addresses: weili96@zju.edu.cn (W. Li). 
Abstracts: Heat transfer and flow characteristics of Taylor flow in vertical capillaries with diameters of $0.5,1$, and $2 \mathrm{~mm}$ have been investigated numerically with the volume of fluid method. 30 different cases have been conducted with inlet Reynolds number ranging from 100 to 500 and initial void fraction ranging from 0.2 to 0.4 . Simulation results of the normalized bubble rising velocity, frictional pressure drops, and average Nusselt number fit well with the experimental data and empirical correlations. The results indicate that the normalized bubble rising velocity is related to the capillary number. The flow-pattern dependent and modified Lockhart-Martinelli correlations can predict the numerical frictional pressure drops well. A low temperature region is formed in the liquid slug, where a recirculation zone is formed. The average Nusselt number increases with increasing $R e$, tube diameters, and with decreasing $\xi_{G}$, and is about 1.2 3 times of that of fully developed laminar single phase flow in circular tubes with constant wall heat flux boundary conditions.

Keywords: Taylor flow, Numerical simulation, Micro-channels, Dynamic mesh model 


\section{NOMENCLATURE}

Bo Bond number, $g\left(\rho_{L}-\rho_{G}\right) d^{2} / \sigma$

C Chisholm parameter

Co Courant number

$c_{p} \quad$ Specific heat capacity, $\mathrm{J} \mathrm{kg}^{-1} \mathrm{~K}^{-1}$

$\mathrm{Ca} \quad$ Capillary number, $\mu_{L} U_{T P} / \sigma$

d Hydrodynamic diameter, $\mathrm{m}$

E Internal energy, $\mathrm{J} \mathrm{kg}^{-1}$

$f \quad$ Frictional factor, $\Delta P d /\left(2 \rho_{L} \beta_{L} V_{t p}{ }^{2} L_{c}\right)$

$g \quad$ Gravity acceleration, $9.81 \mathrm{~m} \mathrm{~s}^{-2}$

$k \quad$ Thermal conductivity, $\mathrm{W} \mathrm{m}^{-1} \mathrm{~K}^{-1}$

$L_{c} \quad$ Length of the computational domain, $m$

$L_{\text {slug }} \quad$ Length of the liquid slug, $\mathrm{m}$

$L_{s}^{*} \quad$ Dimensionless slug length, $L_{s l u g}\left(\operatorname{Pr}_{L} R e_{t p} d\right)$

$\mathrm{Nu} \quad$ Nusselt number

$N u_{L} \quad$ Slug Nusselt number, $N u_{t p} / \beta_{L}$

$N u_{x} \quad$ Local Nusselt number

$N u_{t p} \quad$ Average Nusselt number

$P \quad$ Pressure, $\mathrm{Pa}$

$q_{w} \quad$ Wall heat flux, $\mathrm{W} \mathrm{m}^{-2}$

$r \quad$ Radial coordinate

$R \quad$ Radius of the capillary, $d / 2, \mathrm{~m}$

Re Two-Phase Reynolds number, $V_{T P} \rho_{L} d / \mu_{L}$

$S u_{G} \quad$ Suratman number, $\rho_{G} \sigma d / \mu_{G}{ }^{2}$

$T \quad$ Temperature, $\mathrm{K}$

$v \quad$ Velocity, $\mathrm{m} \mathrm{s}^{-1}$

$v_{X} \quad$ Axis velocity, $\mathrm{m} \mathrm{s}^{-1}$

$V_{b} \quad$ Bubble velocity, $\mathrm{m} \mathrm{s}^{-1}$

$V_{T P} \quad$ Mixture velocity, $\mathrm{m} \mathrm{s}^{-1}$ 
X Lockhart-Martinelli parameter

Greek letters

$\alpha \quad$ Volume fraction

$\beta \quad$ Liquid slug ratio, $L_{\text {slug }} / L_{c}$

$\kappa \quad$ Curvature of the interface, $\mathrm{m}^{-1}$

$\mu \quad$ Dynamic viscosity, $\mathrm{N} \mathrm{m}^{-1}$

$\xi \quad$ Initial gas hold up

$\rho \quad$ Density, $\mathrm{kg} \mathrm{m}^{-3}$

$\sigma \quad$ Surface tension coefficient, $\mathrm{N} \mathrm{m}^{-1}$

$\Phi^{2} \quad$ two-phase pressure drop multiplier

Subscripts

$b$,in Liquid bulk for velocity inlet

b,out Liquid bulk for pressure outlet

cal Results predicted by correlation

$f \quad$ Frictional

L Liquid phase

G Gas phase

sim Results obtained by simulation

tp Two phase, average value for a computational domain

w Wall

$w x \quad$ Local temperature on wall

$x s \quad$ Cross section 


\section{Introduction}

Micro-structured devices have been used in a variety of applications, including micro reactors, catalyst coating in capillaries, flow boiling in heat sinks, micro heat pipes, and refrigeration industry. As the flow in micro channels is mainly laminar, the heat and mass transfer process are determined by the diffusing effect. One possible way to further enhance the heat and mass transfer process is adopting two-phase flow like boiling, condensation, and gas-liquid flow without phase change. Non-boiling slug flow has been applied in numerous situations because of its stability and maneuverability. Taylor flow is a special case of gas-liquid flow where Taylor bubbles are separated by liquid slugs. A very thin liquid film is formed between the Taylor bubble and the wall. The heat and mass transfer process and pressure drop of Taylor flow in micro channel have been investigated experimentally and numerically in recent years, but there still remain some gaps in this field.

A large amount of experimental work on hydrodynamic characteristics of Taylor flow has been conducted, concerning the bubble velocity, liquid film thickness, pressure drops. The bubble velocity is important to determine the heat and mass transfer and pressure drops in Taylor flow. Liu et al. ${ }^{[1]}$ studied the hydrodynamics in vertical capillaries with circular and square cross-sectional shapes with hydraulic diameters ranging from 0.9 to $3 \mathrm{~mm}$. The results showed that the capillary geometry, capillary hydraulic diameter, and fluid properties had slight effects on the bubble rising velocity. The tube inclination also has an important effect on the bubble velocity as pointed out by White and Beardmore ${ }^{[2]}$. Lim et al. ${ }^{[3]}$ conducted an experimental work in horizontal tubes and reported that the bubble velocity was even smaller than the mixture velocity in some cases. Several correlations for the liquid film thickness in Taylor flow had been developed by Irandoust and Andersson ${ }^{[4]}$, Aussillous and Quéré ${ }^{[5]}$, and Han and Shikazono ${ }^{[6]}$. In the work of Aussillous and Quéré ${ }^{[5]}$, the inertia force was negligible for the low $R e$ in the experiment and the 
dimensionless liquid film thickness was determined by the capillary number. However, this correlation may overestimate or underestimate the liquid film thickness as a consequence of inertia effects. Han and Shikazono ${ }^{[6]}$ pointed out that the inertia force cannot be neglected when capillary number is relatively high and they adopted $R e$ and We in their correlation to take the inertia force into account.

Compared with extensive work on hydrodynamic characteristics and mass transfer in Taylor flow, the heat transfer work is limited. Leung et al. ${ }^{[7,8]}$ experimentally investigated the effect of flow characteristics on Taylor flow heat transfer in a $2 \mathrm{~mm}$ vertical tube with $C a$ ranging from 0.001 to 0.18 . Their results showed that the mixture velocity, the film thickness, and recirculation zones have significant effects on the heat transfer coefficients. A correlation to predict the heat transfer rate was developed based on their experimental data. Walsh et al. ${ }^{\text {[9] }}$ investigated heat transfer characteristics of Taylor flow under constant heat flux boundary conditions. Infrared thermograph was used to obtain the local Nusselt number in the entrance and fully developed flow region, and a correlation to predict the local $\mathrm{Nu}$ was proposed. Lim et al. ${ }^{[3]}$ combined the investigation of flow visualization and heat transfer characteristics in a circular tube coated with a thin uniform indium tin oxide. The Nussle number for two-phase bubbly flow was found to increase by $176 \%$ compared with the single phase flow. Maunder et al. ${ }^{[10]}$ studied the local $\mathrm{Nu}$ of gas-liquid Taylor flow in square mini channels and found that the heat transfer coefficient was about 1.2-1.6 times higher. Recently, Bandera et al. ${ }^{[11]}$ made a review about the heat transfer of Taylor flow in microchannels. Five Nusselt number correlations proposed by Oliver and Wright ${ }^{[12]}$, Hughmark ${ }^{[13]}$, Kreutzer et al. ${ }^{[14]}$, Walsh et al. ${ }^{[9]}$, and Leung et al. ${ }^{[7]}$ were analyzed. However, these correlations give widely varying values for identical conditions.

Because of the limitation of measuring points, it is difficult to obtain detailed depictions of the flow field and temperature distribution in Taylor flow using experimental methods. With the development of computational hardware and software, Computational Fluid Dynamics (CFD) has gained popularity in investigating the heat and fluid characteristics of Taylor flow. As mentioned by Talimi et al. ${ }^{[15]}$, the 
computational domain commonly used by researchers could be categorized into two main groups: fixed frames reference (FFR) and moving frames reference (MFR). The application of MFR depends on the accurate initial value of the computational domain length and gas phase void fraction. Compared with the FFR method, the MFR method is more efficient because of the shorter computational domain and less mesh elements. Since the bubble volume must be initially set, the MFR method is incompetent to investigate the formation and collapse of Taylor bubbles.

For the numerical work about Taylor flow, the mesh near the tube wall is significant to obtain the thin liquid film especially at lower $C a$. As pointed by Gupta et al. ${ }^{[16,17]}$, a minimum of five elements were recommended to capture the liquid film near the tube wall. With fewer meshes, the bubble will attach to the tube wall, and the shape of bubble is mainly determined by the contact angle. Similar studies using FFR were conducted by Qian and Lawal [18], Santos and Kawaji ${ }^{[19]}$, Shao et al. ${ }^{[20]}$, Mehdizadeh et al. ${ }^{[21]}$, and Gregorc and Zun ${ }^{[22]}$. However, the liquid film was failed to be captured in the work of Qian and Lawal ${ }^{[18]}$ and Shao et al. ${ }^{[20]}$.

The numerical simulation using moving frame computational domains can also be divided into two types: single-phase and two-phase moving frame simulations. For the single-phase moving frame, the effect of gas phase on momentum equations is neglected. A moving wall boundary condition is adopted to actuate the fluid in liquid slug zone. Kreutzer et al. ${ }^{[23]}$, Talimi et al. ${ }^{[24]}$, and Che et al. ${ }^{[25,26]}$ adopted this method to perform Taylor flow simulations. However, the liquid film thickness was not considered in Talimi et al. ${ }^{[24]}$ and Che et al. ${ }^{[25,26]}$. This led to higher heat transfer coefficients and pressure drops as reported in Talimi et al. ${ }^{[24]}$. The simulation using the two-phase MFR method is carried out in a moving frame of reference attached to Taylor bubbles using the dynamic mesh model or the moving frame method. Zheng et al. ${ }^{[27]}$, Taha and Cui ${ }^{[28,29]}$, and Liu and Wang ${ }^{[30]}$ adopted the moving frame method to studied the Taylor flow in circular and noncircular capillaries. Asadolahi et al. ${ }^{[31,32]}$ investigated hydrodynamic and heat transfer characteristics in Taylor flow using the fixed frame and moving frame methods. A periodic boundary condition was adopted using the User_Defined_Function (UDF) in ANSYS Fluent. This method was 
validated by the high consistency between numerical and experimental results. Che et al. ${ }^{[33]}$ investigated convective heat transfer characteristics of droplet-based micro heat sinks. The level-set method was employed to obtain the two-phase interfaces.

Though numerous work has been done, the characteristics of the fully developed Taylor flow have not been investigated in detail. With the purpose of bridging this research gap, the present study is designed for a further understanding of the fully developed Taylor flow. The two-phase MFR method has been adopted in a 2D, axis symmetrical model to investigate hydraulic and heat transfer characteristics of fully developed Taylor flow in three cylindrical capillaries with diameter of 0.5, 1, $2 \mathrm{~mm}$ at Re ranging from 100 to 500 and $\xi_{G}$ ranging from 0.2 to 0.4 . Numerical results are compared with experimental data and empirical correlations.

\section{Numerical model}

A commercial CFD code [ANSYS Fluent (Release 14.0)] based on finite volume is used to perform the numerical simulation. The volume of fluid (VOF) model is adopted to capture the gas-liquid interface in this simulation. The VOF model accomplishes the interface tracking by solving an additional continuity-like equation for the volume fraction. Two phases are assumed incompressible and not penetrating each other. Compared with other interface capture methods, like Level-set and Phase field, the main advantage of VOF is its inherent volume conserving nature.

\subsection{Governing equations}

A single-fluid formulation is applied throughout the computational domain where velocity and pressure fields are shared amongst two phases. Properties of single-fluid are calculated based on the volume weight fraction of each phase in the cell. A more detailed description of the VOF model could be found in the work presented by Jeon et al. ${ }^{[34]}$. The governing equations in the present investigation are:

Continuity Equation: 


$$
\frac{\partial \rho}{\partial t}+\nabla \cdot(\rho v)=0
$$

Momentum Equation:

$$
\frac{\partial(\rho v)}{\partial t}+\nabla \cdot(\rho v v)=-\nabla P+\nabla \cdot\left[\mu\left(\nabla v+\nabla v^{T}\right)\right]+\rho \mathrm{g}+F
$$

Energy Equation:

$$
\frac{\partial(\rho E)}{\partial t}+\nabla \cdot[v(\rho E+P)]=\nabla \cdot[k(\nabla T)]
$$

Volume Fraction Equation:

$$
\frac{\partial(\alpha)}{\partial t}+v \cdot \nabla \alpha=0
$$

where

$$
\begin{gathered}
\rho=\alpha_{L} \rho_{L}+\alpha_{G} \rho_{G} \\
\mu=\alpha_{L} \mu_{L}+\alpha_{G} \mu_{G} \\
k=\alpha_{L} k_{L}+\alpha_{G} k_{G} \\
\alpha_{L}+\alpha_{G}=1
\end{gathered}
$$

Surface tension is included with the source term $\boldsymbol{F}$ in momentum equation. A continuum surface force (CSF) model proposed by Brackbill et al. ${ }^{[35]}$ is adopted in this work. $\boldsymbol{F}$ is defined as follows:

$$
F=\sigma \frac{\alpha_{L} \rho_{L} \kappa_{G} \nabla \alpha_{G}+\alpha_{G} \rho_{G} \kappa_{L} \nabla \alpha_{L}}{0.5\left(\rho_{L}+\rho_{G}\right)}
$$

\subsection{Computational domain and numerical method}

The geometry of computational domain with a total length of $10 d$ with boundary conditions is shown in Fig. 1. The velocity inlet and pressure outlet boundary conditions are applied in the upstream and downstream. A no-slip boundary condition with a constant heat flux of $32 \mathrm{~kW} \mathrm{~m}^{-2}$ is employed at the wall and a zero normal gradient boundary condition is adopted at the axis for all variables. Constant property nitrogen and water (listed in Table 1) are used as working fluids. 


\section{Table 1}

Properties of the working fluid

\begin{tabular}{cccccc}
\hline Fluid & $\rho\left(\mathrm{kg} \mathrm{m}^{-3}\right)$ & $\mu\left(\mathrm{kg} \mathrm{m}^{-1} \mathrm{~s}^{-1}\right)$ & $k\left(\mathrm{~W} \mathrm{~m}^{-1} \mathrm{~K}^{-1}\right)$ & $c_{p}\left(\mathrm{~J} \mathrm{~kg}^{-1} \mathrm{~K}^{-1}\right)$ & $\sigma\left(\mathrm{N} \mathrm{m}^{-1}\right)$ \\
Nitrogen & 1.145 & $2.1 \times 10^{-5}$ & 0.0242 & 1040 & \\
Water & 997 & $88.99 \times 10^{-5}$ & 0.60 & 4182 & 0.072 \\
\hline
\end{tabular}

Initially, a fully-developed parabolic velocity is set in the whole domain with an average velocity equals to that of the two phase mixture. At the beginning of the simulation, a cylindrical bubble is set in the middle of the computational domain with a volume equals to the product of gas hold-up and unit cell volume. The dynamic mesh model is applied in the present work, and the velocity of moving computational domain equals to the bubble velocity calculated by Eqs. 10 .

$$
V_{b}=\frac{\int_{x}^{x+L c} \int_{0}^{R} \alpha_{G} \nu_{x} 2 \pi r d r d x^{\prime}}{\int_{x}^{x+L c R} \int_{0}^{R} \alpha_{G} 2 \pi r d r d x^{\prime}}
$$

After each iteration, velocity components at the inlet boundary are specified to the value of the pressure outlet in the downstream. Once the steady final bubble is obtained, the flow field is frozen, only leaving the energy equation being solved. The scaled temperature profile is wrapped from faces where flow leaves the domain to the corresponding ones as depicted in Fig. 1. The temperature difference of heat addition at the wall is calculated via Eqs. 11.

$$
\Delta T_{C}=\frac{q_{w} A_{w}}{\left[\left(V_{t p}-\xi_{G} V_{b}\right) \rho_{L} C p_{L}+\xi_{G} V_{b} \rho_{G} C p_{G}\right] A_{x s}}
$$

More detailed description of this method and the derivation of Eqs. 11 can be found in Asadolahi et al. ${ }^{[31]}$. In the present work, the UDF for performing this method is parallelized. For a similar case with Asadolahi et al. ${ }^{[31]}$, it took about 8 hours to get a stable Taylor bubble using 4 processors on a 64 bit PC having 8 GB RAM, less than the single processor work (about 29 hours) in Asadolahi et al. ${ }^{[31]}$. 


\subsection{Numerical simulation parameters}

A first order, non-iterative fractional step scheme is used for the time marching of the continuity, momentum, energy, and volume fraction equations. The implicit body force treatment is used to take the partial equilibrium of pressure gradients and body forces into account. For the momentum and energy discretization, the QUICK scheme has been used. Body-force-weighted and Geo-reconstruct interpolation scheme are used for the discretization of pressure and volume fraction, respectively. A variable time step, controlled by a specified maximum value of Courant number $(\mathrm{Co})$, is used for the momentum and pressure equations. A maximum Co of 0.25 is set in the present study. Co is defined as bellow:

$$
C o=\frac{v \Delta t}{\Delta x}
$$

where $\Delta x$ is the mesh size, and $v$ is the fluid velocity.

\section{Grid independence}

As recommended by Gupta et al. ${ }^{[16]}$, structured square grid, which can enhance the accuracy of surface tension calculation in the gas-liquid interface region, is adopted in the present work. As depicted in Fig. 1, the mesh is refined near the wall. In the present work, 20 elements are set near the wall region to capture the thin liquid film, much higher than 5 elements recommended by Gupta et al. ${ }^{[16]}$. The gas-liquid interface profile using three different grid sizes is shown in Fig. 2. Bubble shapes and liquid film thickness remain unchanged with increasing grid number more than $95 \times 1540$, where 95 represents the element number in radial direction, 1540 for axis direction. The bubble length of $86 \times 1326$ is longer than others, while the liquid film obtained in this mesh is thicker than the other two. 


\section{Results and discussion}

\subsection{The effects of $R e$ and $\xi_{G}$ on bubble shapes and bubble rising velocities}

A global perspective is depicted in Fig. 3 about the influence of $R e$ (constant $\xi_{G}$ of 0.4) and $\xi_{G}$ (constant $R e$ of 500) on bubble shapes, streamlines, and velocity fields in a capillary $(d=0.5 \mathrm{~mm})$. Both stream lines and velocity fields are defined in a moving frame of reference attached to the Taylor bubble. This type of qualitative graphical representation is inspired by Kang et al. ${ }^{[36]}$ and Araújo et al. ${ }^{[37]}$, who provided simulations of Taylor bubble in stagnant liquids. In the present work, Taylor bubble consists of a spherical nose and tail, as well as, a cylindrical gas column. A thin liquid film between the Taylor bubble and the tube wall is formed. The recirculation zone is observed in gas and liquid slugs. This is different from the work of Araújo et al. ${ }^{[37]}$, who obtained a single recirculation zone in the bubble region. Three recirculation zones, a large one in the middle and two small one near the nose and tail, are observed inside the Taylor bubble. The bubble length and recirculation zone increase with increasing $\xi_{G}$. The relative velocity magnitude in the computational domain maintains parabolic except regions near the nose and tail of Taylor bubbles.

A clear and quantitative description of the streamline and velocity field in the capillary tube $(d=0.5 \mathrm{~mm})$ at $R e=300$ is depicted in Fig. 4a. The recirculation zone in liquid region can enhance the heat and mass transfer in Taylor flow. The relative axial velocity in radial direction is illustrated in Fig. 4b. It is obvious that a parabolic velocity is obtained in $x / d=1,5,9$. The relative axial velocity is the same at $x / d=1$ and $x / d=9$ because of the periodic velocity adopted in the present work. The magnitude of axial velocity at $x / d=5$ is higher than that of the inlet and outlet region, while the maximum velocity at $x / d=3$ and 7 is lower than that of the inlet and outlet region. A minimum value is found near the wall region where a small circulation exists at 
$x / d=3$.

A quantitative illustration of the bubble length and liquid film thickness is shown in Fig. 5. The bubble length and liquid film thickness increase with increasing $R e$ at fixed $\xi_{G}$. The unstable zone near the tail of Taylor bubble increases with and the convex shape of Taylor bubble flattens with increasing Re. For capillaries, surface tension and inertia force maintain the shape of bubbles. As Re increases, inertia force increases, leading to a Taylor bubble with flatter tail, sharper nose, and longer bubble length. Araújo et al. ${ }^{[37]}$ pointed out that for a constant Morton number, shapes of Taylor bubbles can change from convex to concave with decreasing surface tension. As shown in Fig. 5, the bubble shape is similar at different $\xi_{G}$, while the length of the Taylor bubble increases with increasing $\xi_{G}$. The length of stable liquid film region decreases with decreasing $\xi_{G}$, which means Taylor flow may change into bubbly flow at smaller $\xi_{G}$.

As mentioned before, the rising velocity of Taylor bubble is higher than the two-phase superficial velocity. Liu et al. ${ }^{[1]}$ experimentally investigated Taylor flow in vertical capillaries and proposed a correlation (Eqs. 13) based on their experimental data.

$$
\frac{V_{b}}{V_{t p}}=\frac{1}{1-0.61 C a^{0.33}}
$$

The present simulation data together with numerical results conducted by Asadolahi et al. ${ }^{[32]}$, and experimental data from Liu et al. ${ }^{[1]}$ are illustrated in Fig. 6. Eqs. 13 can predict the trend of all data well, and nearly all data locate within the $\pm 5 \%$ error band.

\subsection{Frictional characteristics of Taylor flow at different $R e$ and $\xi_{G}$}

The pressure drop of Taylor flow consists of pressure drops in the liquid slug and in the Taylor bubble region. As shown in Fig. 7a, the friction factor $f$, defined in Eqs. 14, decreases with increasing $R e$, and the product of $f$ and $R e$ for Taylor flow is higher 
than that of single phase flow.

$$
\begin{aligned}
& f=\frac{\Delta P d}{2 L_{c} \rho_{L} V_{t p}^{2} \beta_{L}} \\
& R e=\rho_{L} V_{t p} d / \mu_{L}
\end{aligned}
$$

$f$ also increases with increasing tube diameters, while the difference of $f$ obtained at various tube diameters decreases with increases in Re. Fig. 7b depicted that $f$ increases with increasing $\xi_{G}$ for all three tubes, which means at the fixed cell length, tube diameters, and velocity inlet, $f$ increases with increasing Taylor bubble lengths.

The pressure gradients correlations for Taylor flow can be categorized into two main groups: the flow-pattern related model, which is strongly depend on the accurate prediction of the liquid and gas slug lengths, and the separate model (listed in Table 2) proposed by Lockhart and Martinelli ${ }^{[38]}$, which is widely used in predicting pressure drops for boiling and condensation cases.

According to Kreutzer et al. ${ }^{[23]}$, for a given liquid property and tube geometry parameters, $f R e$ is determined by the dimensionless liquid length $d / L_{\text {slug }}(\operatorname{Re} / \mathrm{Ca})^{0.33}$. It should be noted that the effect of capillary angle hysteresis and the pressure drop caused by deformation of bubble surface were not included in this model. Warnier et al. ${ }^{[39]}$ took the gas bubble velocity into account and presented a model for predicting pressure drops in the gas-liquid Taylor flow. Compared with Kreutzer et al. ${ }^{[23]}$, this model shows better agreement with the experimenta work when $R e_{L}$ is smaller than 150. Abiev ${ }^{[40]}$ developed a mathematical model to describe the pressure losses for the slug flow in mini and micro channels. The rearrangement of the velocity profile in the liquid slug and energy losses on the formation and renewal of interfacial area were taken into account. He also pointed that pressure losses were detemined by the number of Taylor bubbles rather than the gas holdup. Jovanovic et al. ${ }^{[41]}$ investigated the hydrodynamics and pressure drops in the liquid-liquid slug flow and presented a pressure drop model. The pressure drop for the dispersed phase, continuous phase, and interfaces were employed in their model. The descriptions of these correlations can be found in Table 2. 
Though the Lockhart-Martinelli correlation is not developed specially for gas-liquid Taylor flow, it is still adopted for comparison with our numerical work because of its convenient application compared with the flow-pattern related models mentioned before. Lim et al. ${ }^{[3]}$ adopted the Lockhart-Martinelli correlation to predict their experimental pressure drops in capillaries with diameters of 0.5 and $0.3 \mathrm{~mm}$. He pointed that the Chisholm number $C=5$, which was used for macro channels, would underestimate pressure drop in micro channels. Qu and Mudawar ${ }^{[42]}$ and Lee and Lee ${ }^{[43]}$ revised $C$ in the Lockhart-Martinelli correlation to predict their experimental data. $\mathrm{Li}$ (corresponding author) and $\mathrm{Wu}{ }^{[44]}$ and Kim and Mudawar ${ }^{[45]}$ analyzed the experimental data of adiabatic pressure drop in micro channels obtained from open literatures and proposed a modified model taking the surface tension, tube diameters, and mass flux into account. More information about these correlations can be found in Table 2.

\section{Table 2}

Description of correlations for pressure drop gradients

$$
\begin{aligned}
& \text { Authors Equations } \\
& \text { Jovanovic et al. }{ }^{[41]} \quad(-d P / d x) f=\left(\frac{8 V_{b} \mu_{g} L_{b}}{R_{b}^{2} L_{c}}+\frac{8 V_{t p} \mu_{L} L_{L}}{R^{2} L_{c}}+\frac{7.16(3 C a)^{2 / 3} \sigma}{d L_{c}}\right) \\
& \text { Abiev }{ }^{[40]} \\
& a=\frac{8 \mu_{L} V_{t p}}{R^{2}} \quad b=\frac{\mu_{L} \gamma_{m}^{2}}{\rho_{L} V_{t p} R^{2}} \\
& (-d P / d x) f=\frac{a L_{L}}{L_{c}}\left\{1+\frac{1}{2 b L_{L}}\left[1-\exp \left(-b L_{L}\right)\right]\right\}+\frac{2 R_{b}\left(V_{b}-V_{t p}\right) \sigma}{R^{2} V_{t p} L_{c}} \\
& (-d P / d x)_{f}=\frac{32}{\operatorname{Re}_{t p}}\left[1+0.07 \frac{d}{L_{\text {slug }}}\left(\frac{\mathrm{Re}}{C a}\right)^{0.33}\right] \frac{\beta_{L} \rho_{L} V_{t p}^{2}}{d} \\
& (-d P / d x)_{f}=\frac{32 \mu_{L} V_{L}}{d^{2}}\left[1+\frac{7.16 \times 3^{2 / 3}}{32} \frac{d f_{b}}{V_{L}\left(C a_{b}^{1 / 3}+3.34 C a_{b}\right)}\right]
\end{aligned}
$$

Lockhart and

Martinelli

$$
(-d P / d x)_{f}=(-d P / d x)_{L} \Phi_{L}^{2}
$$


correlation $^{[38]}$

$$
\begin{gathered}
\Phi_{L}^{2}=1+C / X_{t t}+1 / X_{t t}^{2} \quad X_{t t}^{2}=(-d P / d x)_{L} /(-d P / d x)_{G} \\
(-d P / d x)_{L}=2 f_{L} V_{L}^{2} /\left(d \rho_{L}\right) \quad(-d P / d x)_{G}=2 f_{G} V_{G}^{2} /\left(d \rho_{G}\right) \\
\operatorname{Re}<2000 \quad f=16 / \operatorname{Re} \\
\text { For Bo } \leq 1.5, \quad C=11.9 B o^{0.45}
\end{gathered}
$$

$\mathrm{Li}$ and $\mathrm{Wu}{ }^{[44]}$

$$
\text { For } 1.5<\text { Bo } \leq 11, \quad C=109.4\left(B o \mathrm{Re}_{L}^{0.5}\right)^{-0.56}
$$

Kim and Mudawar

$$
C=3.5 \times 10^{-5} \operatorname{Re}_{L O}^{0.44} S u_{G O}^{0.50}\left(\rho_{L} / \rho_{G}\right)^{0.48}
$$

$$
S u_{G O}=\rho_{G} \sigma d / \mu_{G}^{2}
$$

Qu and Mudawar

$$
C=21\left[1-\exp \left(-319 d_{h}\right)\right](0.00418 G+0.0613)
$$

$$
\text { Lee and Lee }{ }^{[43]} \quad C=6.833 \times 10^{-8} d^{1.874} V_{t p}^{0.719} V_{L O}^{0.557} \mu_{L}^{-2.472} \rho_{L}^{1.874} \sigma^{0.598}
$$

The comparisons between simulation data and calculated results obtained by eight pressure drop correlations are illustrated in Fig. 9. Except for the Lee and Lee ${ }^{\text {[43] }}$ model, all these correlations can predict our numerical data within the $\pm 20 \%$ error band. Both the flow-pattern related model and the modified Lockhart-Martinelli correlation are capable to estimate the numerical pressure drop gradients in the present work. For better comparison of these models, the mean absolute deviation (MAD) and the mean relative deviation (MRD) values which are calculated in Eqs. 16 and Eqs. 17, respectively, are adopted.

$$
\begin{gathered}
M A D=\frac{1}{N} \sum_{1}^{N} \frac{a b s\left(U_{\text {pre }}-U_{\text {sim }}\right)}{U_{\text {sim }}} \bullet 100 \\
M R D=\frac{1}{N} \sum_{1}^{N} \frac{U_{\text {pre }}-U_{\text {sim }}}{U_{\text {sim }}} \bullet 100
\end{gathered}
$$

where $U$ means the value of a numerical or calculated data point.

As listed in Table 3, the Kreutzer et al. ${ }^{[23]}$ correlation can predict the numerical data best compared with other correlations with $\mathrm{MAD}=5.92 \%$ and $\mathrm{MRD}=-0.7 \%$ for all tubes, respectively. Compared with other three modified Lockhart-Martinelli correlations, the Kim and Mudawar ${ }^{[45]}$ correlation shows better comparison results 
with numerical data with $\mathrm{MAD}=9.55 \%$ and $\mathrm{MRD}=-1.16 \%$ for all tubes, respectively.

Table 3

MAD and MRD calculated for different correlations with different tube diameters

\begin{tabular}{|c|c|c|c|c|c|c|c|c|}
\hline \multirow{2}{*}{ Correlations } & \multicolumn{2}{|c|}{$\mathrm{d}=0.5 \mathrm{~mm}$} & \multicolumn{2}{|c|}{$\mathrm{d}=1 \mathrm{~mm}$} & \multicolumn{2}{|c|}{$\mathrm{d}=2 \mathrm{~mm}$} & \multicolumn{2}{|c|}{ All cases } \\
\hline & MAD & MRD & MAD & MRD & MAD & MRD & MAD & MRD \\
\hline $\begin{array}{l}\text { Jovanovic et al. } \\
\text { [41] }\end{array}$ & 8.29 & -8.29 & 5.05 & -4.89 & 6.97 & -6.29 & 6.86 & -6.29 \\
\hline Abiev $^{[40]}$ & 7.71 & -4.02 & 4.59 & -1.94 & 7.83 & 4.55 & 6.81 & -0.40 \\
\hline Kreutzer et al. ${ }^{[23]}$ & 3.15 & -2.74 & 4.70 & 0.71 & 7.94 & 0.12 & 5.29 & -0.70 \\
\hline Warnier et al. ${ }^{\text {[39] }}$ & 8.47 & -8.47 & 4.89 & -4.71 & 6.50 & -5.69 & 6.71 & -6.36 \\
\hline $\mathrm{Li}$ and $\mathrm{Wu}{ }^{[44]}$ & 9.58 & -9.58 & 8.82 & 6.57 & 18.32 & 16.78 & 12.24 & 11.07 \\
\hline $\begin{array}{l}\text { Kim and } \\
\text { Mudawar }^{[45]}\end{array}$ & 7.60 & -7.60 & 6.23 & -0.14 & 14.81 & 4.25 & 9.55 & -1.16 \\
\hline $\begin{array}{l}\text { Qu and Mudawar } \\
\text { [42] }\end{array}$ & 15.65 & 11.18 & 14.37 & 6.97 & 12.03 & -4.70 & 14.02 & 4.48 \\
\hline Lee and Lee ${ }^{[43]}$ & 32.83 & -32.83 & 34.42 & -34.42 & 39.88 & -39.88 & 35.71 & -35.71 \\
\hline
\end{tabular}

\subsection{Heat transfer characteristics of gas-liquid Taylor flow}

The axial variation of the local wall and bulk temperature together with local Nusselt number $\left(\mathrm{Nu}_{x}\right.$, defined in Eqs. 18) are shown in Fig. 9a. The bulk temperature at each axis location is calculated using the enthalpy weighted method as depicted in Eqs. 19.

where

$$
\begin{gathered}
N u_{x}=\frac{q_{W}}{T_{W}-T_{b x}} \frac{d}{k_{L}} \\
T_{b x}=\frac{\int_{0}^{R} \rho\left|v_{x}\right| c_{p} T(2 \pi r) d r}{\int_{0}^{R} \rho\left|v_{x}\right| c_{p}(2 \pi r) d r} \\
T_{w}=\int_{0}^{L_{c}} T_{w x} d x
\end{gathered}
$$


As illustrated in Fig. 9a, $T_{w}$ increases with $x / d$ in the liquid region and obtains its maximum value at the tail of Taylor bubbles, while $T_{b x}$ decreases along the axis direction in the liquid slug region. In the Taylor bubble region, $T_{w}$ and $T_{b x}$ decrease with $x / d$, and $T_{b x}$ is much higher than that in the liquid region because of the lower heat capacity of the gas phase. In the present work, a constant heat flux condition is adopted on the wall, and $N u_{x}$ is determined by the difference of $T_{w}$ and $T_{b x}$. As shown in Fig. 9a, $N u_{x}$ is higher in the Taylor bubble region than that obtained in the liquid slug region. $N u_{x}$ gets its maximum value at the tail of Taylor bubble. The vortex near the bubble tail leads to a slight vibration of $N u_{x}$.

Fig. 9b shows the contour of temperature in the computational domain along with the bubble shape and streamlines. A low temperature region is formed before and after the Taylor bubble between the wall and axis region. As explained before, the lower heat capacity of gas leads to a higher temperature in the bubble region indicating that heat tends to transfer from the gas bubble to the adjacent liquid slugs. As shown in Fig. 9a, the heat transfer coefficients is much higher in the liquid film region, where heat is much easier to be transferred from the wall to the liquid film and Taylor bubble. The hot fluid which was previously in contact with the wall is brought to the center of the tube and the fluid between the wall and the center remains relatively cold. This shows the effect of internal recirculation in the liquid slug region on heat transfer process in Taylor flow.

Since the local Nusset number provides a poor representation of the heat transfer behavior, an average $\mathrm{Nu}$ calculated by Eqs. 21 is adopted to investigate heat transfer characteristics of Taylor flow.

where $\quad T_{b, i n}=\frac{\int_{0}^{R} \rho\left|v_{i n, r}\right| c_{p} T(2 \pi r) d r}{\int_{0}^{R} \rho\left|v_{i n, r}\right| c_{p}(2 \pi r) d r}$

As depicted in Fig. 10a, $N u_{t p}$ increases with increasing $R e$ and tube diameter, 
while it decreases with increasing $\xi_{G}$. $N u_{t p}$ decreases with the liquid slug length, which is well accordance with the conclusion proposed by Gupta et al. ${ }^{[16]}$ that Taylor bubble contributes very little to the heat transfer process compared with the liquid slug. $N u_{t p}$ is about 1.2 3.8 times higher than that of single phase, which is 4.364 for the fully developed laminar flow in circular tubes with constant wall heat flux conditions.

The correlation proposed by Leung et al. ${ }^{[7]}$ is defined in Eqs. 23.

where

$$
\begin{gathered}
N u_{L}=4.364+0.29 /\left(L_{s}^{*}+0.15 L_{s}^{* 1 / 3}\right) \\
N u_{L}=N u_{t p} / \beta_{L} \\
L_{s}^{*}=L_{s} /\left(\operatorname{Re}_{t p} \operatorname{Pr} d\right)
\end{gathered}
$$

The contribution of Taylor bubble on heat transfer can be found in the second term of the correlation. Our simulation data together with the data obtained by Leung et al. ${ }^{[7]}$ and Lakehal et al. ${ }^{[46]}$ are depicted in Fig. 10b. $N u_{L}$ decreases with increasing dimensionless slug length, and obtains the value of 4.36 when taking the limit for $L_{s}^{*} \rightarrow \infty$. Our simulation data falls within $\pm 20 \%$ error band of Eqs. 23, which is precisely the same as that found by Leung et al ${ }^{[7]}$.

\section{Conclusion}

The hydrodynamic and heat transfer characteristics of water/nitrogen Taylor flow in circular vertical capillaries with diameter of $0.5,1$, and $2 \mathrm{~mm}$ have been investigated numerically using the dynamic mesh model. The following conclusions can be drawn from the present work.

(1) The liquid film thickness and the bubble length increase with increasing $R e$, while the increase of $\xi_{G}$ only changes the length of Taylor bubble and inner recirculation region. Three recirculation zones in the Taylor bubble and two recirculation zones in the liquid slug are observed in Taylor flow. Except the region near the nose and tail, the axis velocity profile in computational domain is parabolic in both gas and liquid slug. The normalized bubble rising velocity $V_{b} / V_{t p}$ is relative to 
$C a^{0.33}$, and Eqs. 13 proposed by Liu et al. ${ }^{[1]}$ can predict our simulation data well.

(2) The friction factor $f$ increases with increasing tube diameters, $\xi_{G}$, and with decreasing $R e$ for all three tubes. Four flow-pattern related pressure drop correlations and four modified Lockhart-Martinelli correlations are adopted to predict the present numerical data, and the former ones show better agreement with numerical results. The Kreutzer et al. ${ }^{[23]}$ correlation can predict the numerical data best compared with other correlations with $\mathrm{MAD}=5.92 \%$ and $\mathrm{MRD}=-0.7 \%$ for all tubes, respectively.

(3) A low temperature region is formed in liquid slug between the wall and axis, where a recirculation zone is formed. Local Nusselt number $N u_{x}$ decreases with $x / d$ in liquid slug region. The internal recirculation in the liquid slug region can enhance heat transfer process in Taylor flow. The average Nusselt number $N u_{t p}$ increases with increasing $R e$ and tube diameters, but decreases with increasing $\xi_{G}$. The numerical data fits well with the empirical correlation developed by Leung et al. ${ }^{[7]} . N u_{t p}$ is about 1.2 3 times higher than the fully developed single-phase laminar flow with constant wall heat flux boundary conditions.

\section{Acknowledgements}

This work is supported by National Science Foundation of China (51210011) and National Science Foundation of Zhejiang Province (Z13E060001). We gratefully acknowledge Prof. David F. Fletcher from the University of Sydney for his kindly and exhaustive help on our simulation works and providing the setup and UDFs that form the basis of this work. We would like to dedicate the paper to commemorate Dr. Ralph L. Webb for his outstanding contributions to heat transfer. Wei Li obtained his Ph.D. with Dr. Webb at Penn State in 1998.

\section{Reference}

[1] H. Liu, C.O. Vandu, R. Krishna, Hydrodynamics of Taylor flow in vertical capillaries: Flow regimes, bubble rise velocity, liquid slug length, and pressure drop, 
Ind. Eng. Chem. Res. 44 (2005) 4884-4897.

[2] E.T. White, R.H. Beardmore, The velocity of rise of single cylindrical air bubbles through liquids contained in vertical tubes, Chem. Eng. Sci. 17 (1962) 351-361.

[3] Y.S. Lim, S.C.M. Yu, N.T. Nguyen, Flow visualization and heat transfer characteristics of gas-liquid two-phase flow in microtube under constant heat flux at wall, Int. J. Heat Mass Transf. 56 (2013) 350-359.

[4] S. Irandoust, B. Andersson, Liquid-film in taylor flow through a capillary, Ind. Eng. Chem. Res. 28 (1989) 1684-1688.

[5] P. Aussillous, D. Quéré, Quick deposition of a fluid on the wall of a tube, Phys. Fluids 12 (2000) 2367-2371.

[6] Y. Han, N. Shikazono, Measurement of the liquid film thickness in micro tube slug flow, Int. J. Heat Fluid Flow 30 (2009) 842-853.

[7] S.S.Y. Leung, Y. Liu, D.F. Fletcher, B.S. Haynes, Heat transfer in well-characterised Taylor flow, Chem. Eng. Sci. 65 (2010) 6379-6388.

[8] S.S. Leung, R. Gupta, D.F. Fletcher, B.S. Haynes, Effect of flow characteristics on Taylor flow heat transfer, Ind. Eng. Chem. Res. 51 (2011) 2010-2020.

[9] P. Walsh, E. Walsh, Y. Muzychka, Laminar Slug Flow: Heat Transfer Characteristics With Constant Heat Flux Boundary, ASME 2009 Heat Transfer Summer Conference collocated with the InterPACK09 and 3rd Energy Sustainability Conferences, American Society of Mechanical Engineers, San Francisco, CA, 2009, pp. 389-397.

[10] A. Majumder, B. Mehta, S. Khandekar, Local Nusselt number enhancement during gas-liquid Taylor bubble flow in a square mini-channel: An experimental study, Int. J. Therm. Sci. 66 (2013) 8-18.

[11] T. Bandara, N.T. Nguyen, G. Rosengarten, Slug flow heat transfer without phase change in microchannels: A review, Chem. Eng. Sci. 126 (2015) 283-295.

[12] D.R. Oliver, S.J. Wright, Pressure drop and heat transfer in gas-liquid slug flow in horizontal tubes, British Chemical Engineering, 9 (1964) 590-596. 
[13] G.A. Hughmark, Holdup and heat transfer in horizontal slug gas-liquid flow, Chem. Eng. Sci. 20 (1965) 1007-1010.

[14] M.T. Kreutzer, P. Du, J.J. Heiszwolf, F. Kapteijn, J.A. Moulijn, Mass transfer characteristics of three-phase monolith reactors, Chem. Eng. Sci. 56 (2001) 6015-6023.

[15] V. Talimi, Y.S. Muzychka, S. Kocabiyik, A review on numerical studies of slug flow hydrodynamics and heat transfer in microtubes and microchannels, Int. J. Multiphase Flow 39 (2012) 88-104.

[16] R. Gupta, D.F. Fletcher, B.S. Haynes, On the CFD modelling of Taylor flow in microchannels, Chem. Eng. Sci. 64 (2009) 2941-2950.

[17] R. Gupta, D.F. Fletcher, B.S. Haynes, CFD modelling of flow and heat transfer in the Taylor flow regime, Chem. Eng. Sci. 65 (2010) 2094-2107.

[18] D. Qian, A. Lawal, Numerical study on gas and liquid slugs for Taylor flow in a T-junction microchannel, Chem. Eng. Sci. 61 (2006) 7609-7625.

[19] R.M. Santos, M. Kawaji, Numerical modeling and experimental investigation of gas-liquid slug formation in a microchannel T-junction, Int. J. Multiphase Flow 36 (2010) 314-323.

[20] N. Shao, W. Salman, A. Gavriilidis, P. Angeli, CFD simulations of the effect of inlet conditions on Taylor flow formation, Int. J. Heat Fluid Flow 29 (2008) $1603-1611$.

[21] A. Mehdizadeh, S.A. Sherif, W.E. Lear, Numerical simulation of thermofluid characteristics of two-phase slug flow in microchannels, Int. J. Heat Mass Transf. 54 (2011) 3457-3465.

[22] J. Gregorc, I. Zun, Inlet conditions effect on bubble to slug flow transition in mini-channels, Chem. Eng. Sci. 102 (2013) 106-120.

[23] M.T. Kreutzer, F. Kapteijn, J.A. Moulijn, C.R. Kleijn, J.J. Heiszwolf, Inertial and interfacial effects on pressure drop of Taylor flow in capillaries, AlChE J. 51 (2005) 2428-2440.

[24] V. Talimi, Y.S. Muzychka, S. Kocabiyik, Numerical simulation of the pressure drop and heat transfer of two phase slug flows in microtubes using moving 
frame of reference technique, Int. J. Heat Mass Transf. 55 (2012) 6463-6472.

[25] Z. Che, T.N. Wong, N.-T. Nguyen, Heat transfer in plug flow in cylindrical microcapillaries with constant surface heat flux, Int. J. Therm. Sci. 64 (2013) 204-212.

[26] Z.Z. Che, T.N. Wong, N.T. Nguyen, Heat transfer enhancement by recirculating flow within liquid plugs in microchannels, Int. J. Heat Mass Transf. 55 (2012) 1947-1956.

[27] D. Zheng, X. He, D. Che, CFD simulations of hydrodynamic characteristics in a gas-liquid vertical upward slug flow, Int. J. Heat Mass Transf. 50 (2007) 4151-4165.

[28] T. Taha, Z.F. Cui, CFD modelling of slug flow in vertical tubes, Chem. Eng. Sci. 61 (2006) 676-687.

[29] T. Taha, Z.F. Cui, CFD modelling of slug flow inside square capillaries, Chem. Eng. Sci. 61 (2006) 665-675.

[30] D. Liu, S. Wang, Hydrodynamics of Taylor flow in noncircular capillaries, Chem. Eng. Process. 47 (2008) 2098-2106.

[31] A.N. Asadolahi, R. Gupta, D.F. Fletcher, B.S. Haynes, CFD approaches for the simulation of hydrodynamics and heat transfer in Taylor flow, Chem. Eng. Sci. 66 (2011) 5575-5584.

[32] A.N. Asadolahi, R. Gupta, S.S.Y. Leung, D.F. Fletcher, B.S. Haynes, Validation of a CFD model of Taylor flow hydrodynamics and heat transfer, Chem. Eng. Sci. 69 (2012) 541-552.

[33] Z. Che, T.N. Wong, N.T. Nguyen, C. Yang, Three dimensional features of convective heat transfer in droplet-based microchannel heat sinks, Int. J. Heat Mass Transf. 86 (2015) 455-464.

[34] S.S. Jeon, S.J. Kim, G.C. Park, Numerical study of condensing bubble in subcooled boiling flow using volume of fluid model, Chem. Eng. Sci. 66 (2011) 5899-5909.

[35] J.U. Brackbill, D.B. Kothe, C. Zemach, A continuum method for modeling surface-tension, J. Comput. Phys. 100 (1992) 335-354. 
[36] C.W. Kang, S.P. Quan, J. Lou, Numerical study of a Taylor bubble rising in stagnant liquids, Phys. Rev. E 81 (2010) 066308-1-11.

[37] J.D.P. Araújo, J.M. Miranda, A.M.F.R. Pinto, J.B.L.M. Campos, Wide-ranging survey on the laminar flow of individual Taylor bubbles rising through stagnant Newtonian liquids, Int. J. Multiphase Flow 43 (2012) 131-148.

[38] R.W. Lockhart, R.C. Martinelli, Proposed correlation of data for isothermal two-phase, two-component flow in pipes, Chem. Eng. Process. 45 (1949) 39-48.

[39] M.J.F. Warnier, M. de Croon, E.V. Rebrov, J.C. Schouten, Pressure drop of gas-liquid Taylor flow in round micro-capillaries for low to intermediate Reynolds numbers, Microfluid Nanofluid, 8 (2010) 33-45.

[40] R.S. Abiev, Modeling of pressure losses for the slug flow of a gas-liquid mixture in mini and microchannels, Theor. Found. Chem. Eng. 45 (2011) 156-163.

[41] J. Jovanovic, W.Y. Zhou, E.V. Rebrov, T.A. Nijhuis, V. Hessel, J.C. Schouten, Liquid-liquid slug flow: Hydrodynamics and pressure drop, Chem. Eng. Sci. 66 (2011) 42-54.

[42] W.L. Qu, I. Mudawar, Measurement and prediction of pressure drop in two-phase micro-channel heat sinks, Int. J. Heat Mass Transf. 46 (2003) 2737-2753.

[43] H.J. Lee, S.Y. Lee, Pressure drop correlations for two-phase flow within horizontal rectangular channels with small heights, Int. J. Multiphase Flow 27 (2001) 783-796.

[44] W. Li, Z. Wu, A general correlation for adiabatic two-phase pressure drop in micro/mini-channels, Int. J. Heat Mass Transf. 53 (2010) 2732-2739.

[45] S. Kim, I. Mudawar, Universal approach to predicting two-phase frictional pressure drop for adiabatic and condensing mini/micro-channel flows, Int. J. Heat Mass Transf. 55 (2012) 3246-3261.

[46] D. Lakehal, G. Larrignon, C. Narayanan, Computational heat transfer and two-phase flow topology in miniature tubes, Microfluid Nanofluid, 4 (2008) 261-271. 


$$
T_{x, r}\left|x+L_{c}=T_{x, r}\right| x+\Delta T_{c}
$$

Dynamic mesh velocity $=V_{b}$

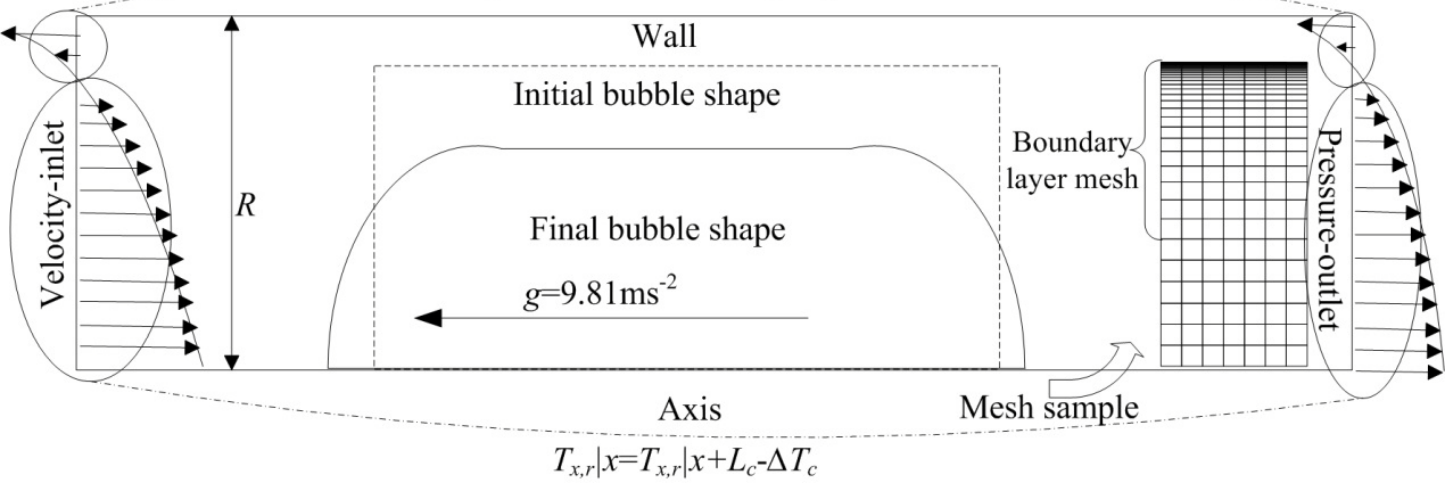

Fig. 1. Computational domain and boundary conditions

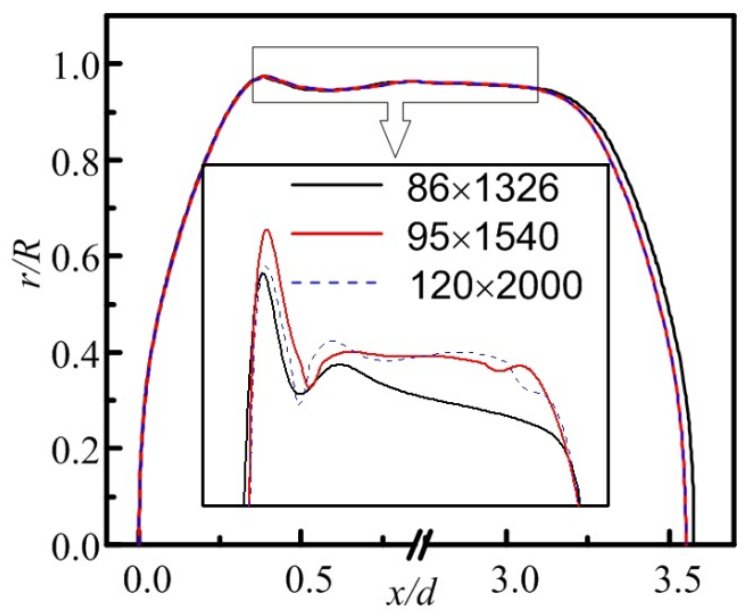

Fig. 2. Taylor bubble shapes for different mesh sizes 

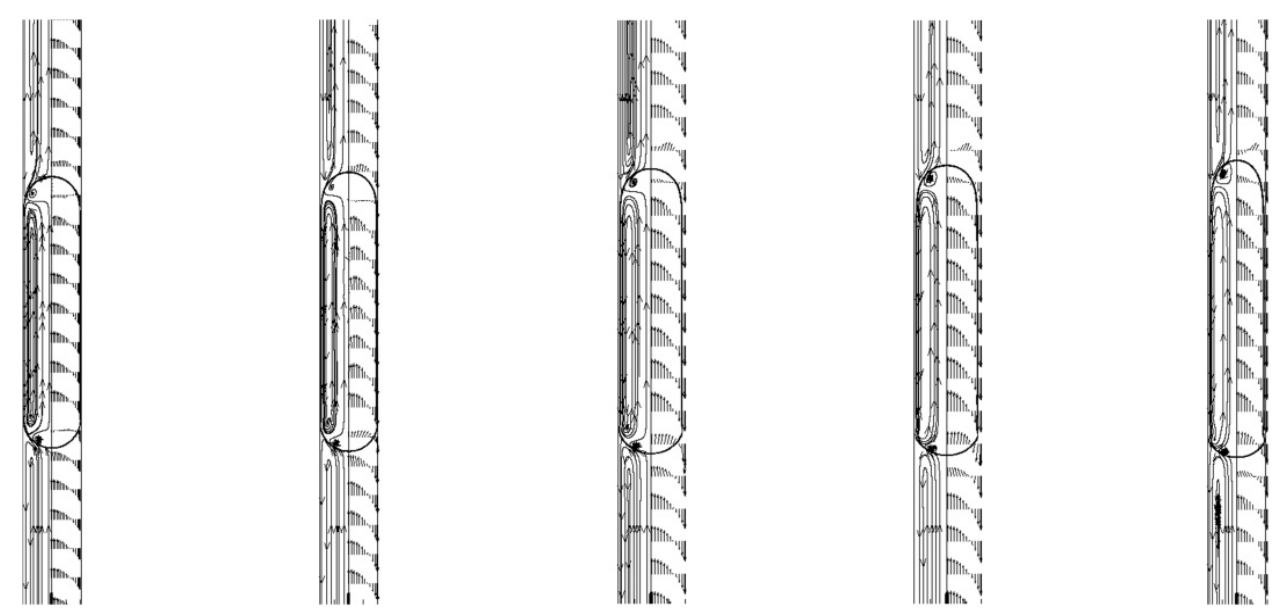

(a) $R e=100$

(b) $R e=200$

(c) $R e=300$

(d) $R e=400$

(e) $R e=500$
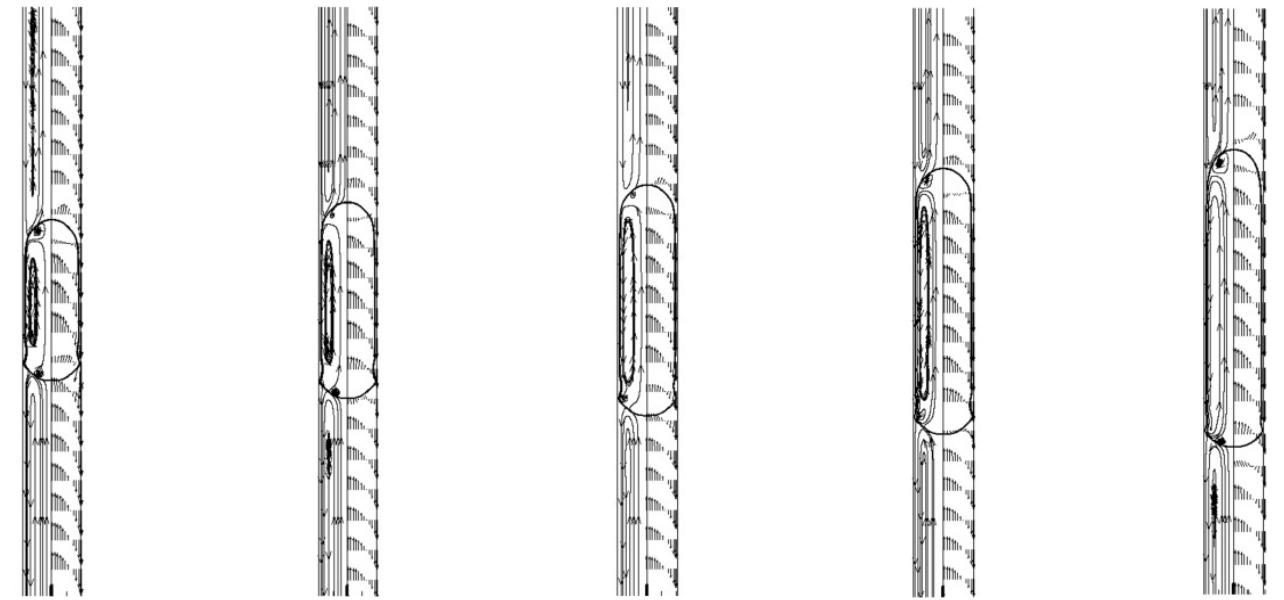

(f) $\xi_{G}=0.2$

(g) $\xi_{G}=0.25$

(h) $\xi_{G}=0.3$

(i) $\xi_{G}=0.35$

(j) $\xi_{G}=0.4$

Fig. 3. Shapes, steam lines, and velocity vectors of Taylor bubble with tube diameter of $0.5 \mathrm{~mm}$ under different conditions ( $\left.R e=100 \sim 500, \xi_{G}=0.2 \sim 0.4\right)$ 


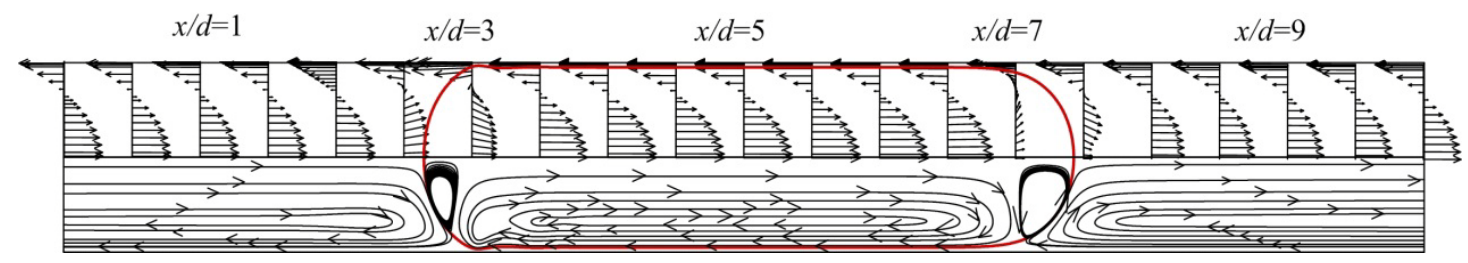

(a)

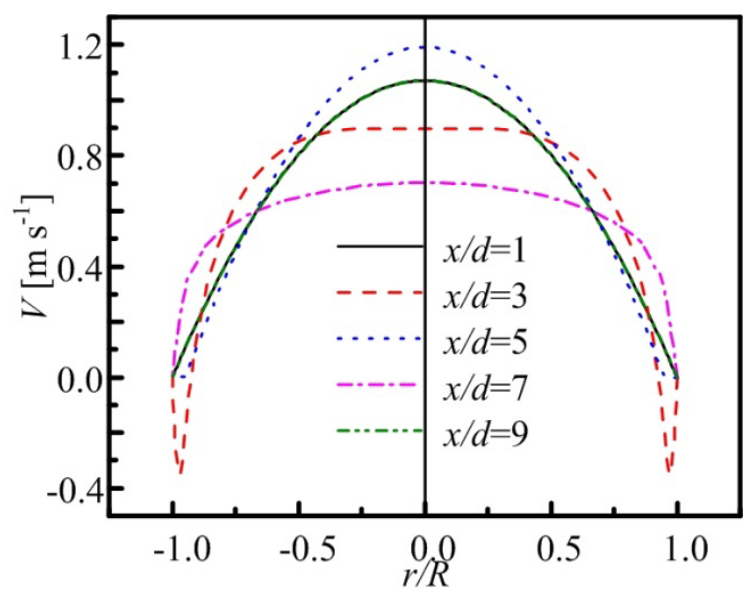

(b)

Fig. 4. (a) Velocity fields and streamlines (b) Relative velocity profile at different $x / d$ for $0.5 \mathrm{~mm}$ capillary at $R e=300$ and $\xi_{G}=0.4$

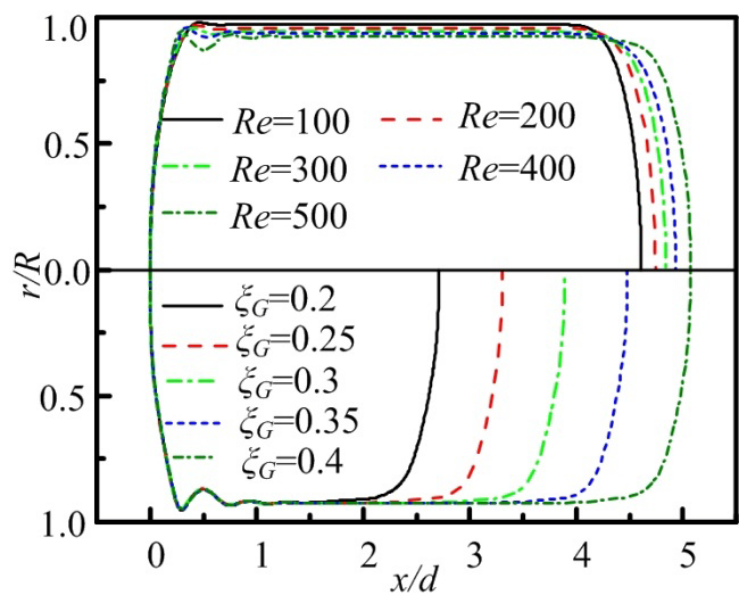

Fig. 5. Taylor bubble shapes for the $0.5 \mathrm{~mm}$ capillary at different $R e$ and $\xi_{G}$ 


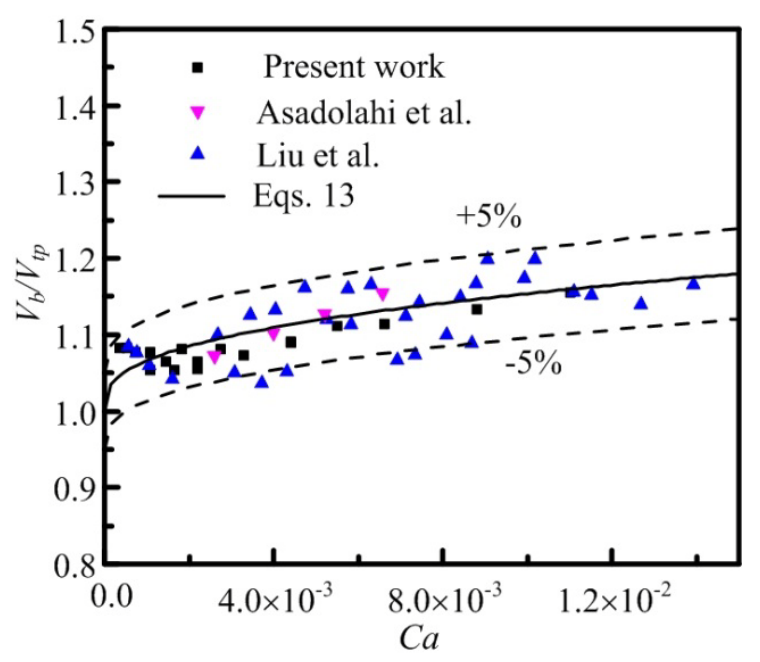

Fig. 6. Effect of $C a$ on $V_{b} / V_{t p}$

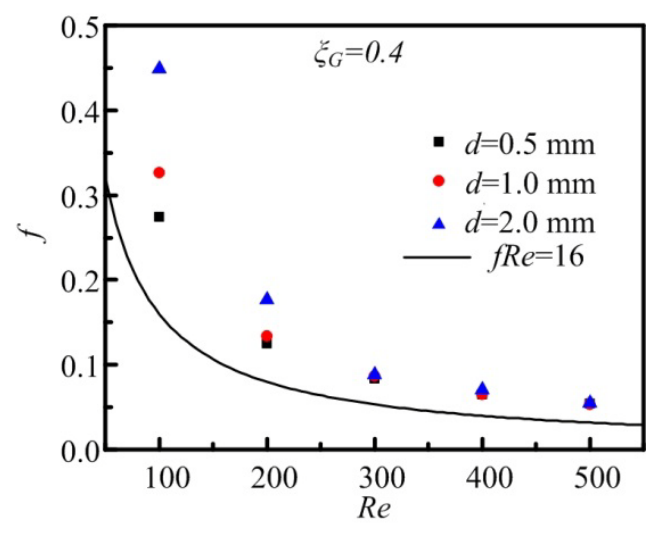

(a)

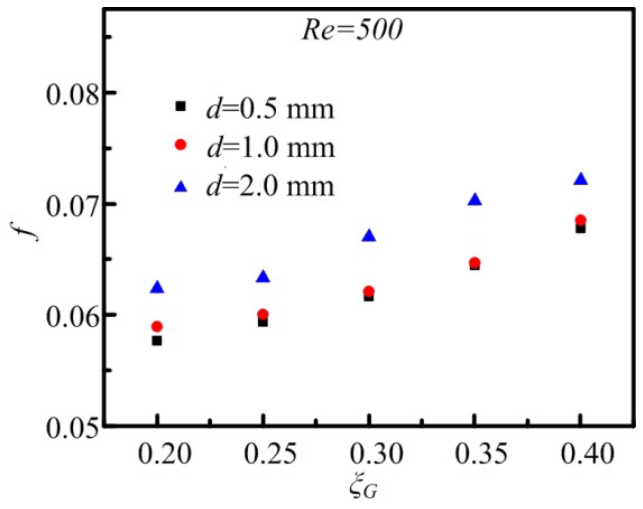

(b)

Fig. 7. The effects of $\operatorname{Re}(\mathrm{a})$ and $\xi_{G}$ (b) on frictional factor $f$

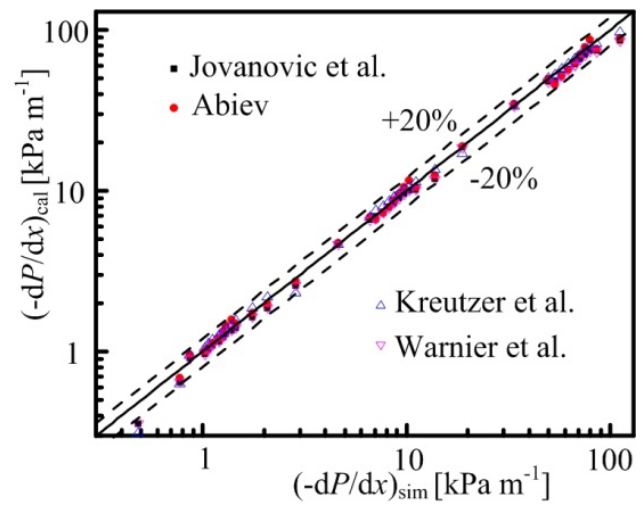

(a)

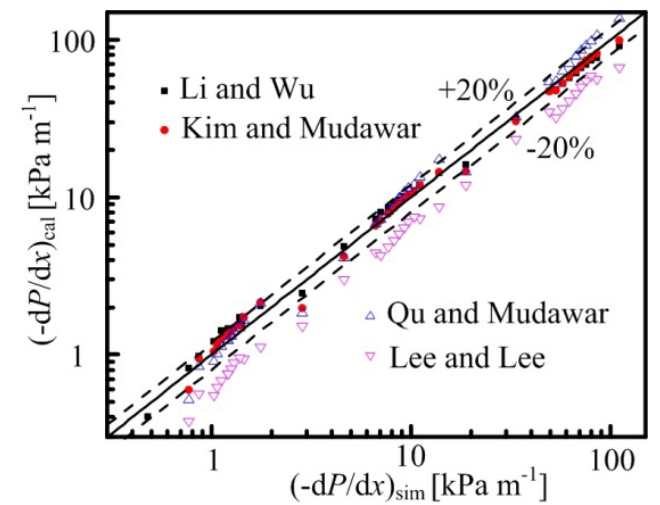

(b)

Fig. 8. Comparison of pressure drop gradients with predictions from (a) flow-pattern related models (b) modified Lockhart-Martinelli correlations $f$ 


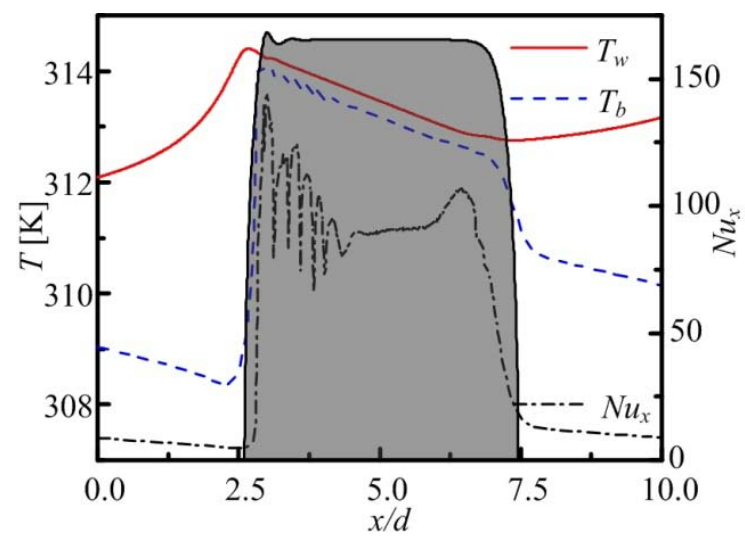

(a)

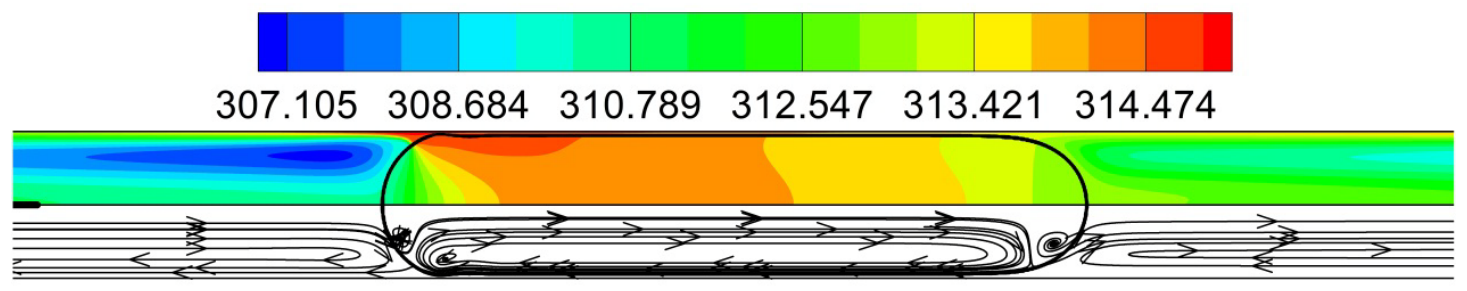

(b)

Fig. 9. (a) Local Nusselt number $\left(N u_{x}\right)$, wall temperature $\left(T_{w}\right)$, liquid bulk temperature $\left(T_{b x}\right)$, and the bubble shape (b) Temperature contours, streamlines, and bubble shape for $0.5 \mathrm{~mm}$ capillary at $R e=300$ and $\xi_{G}=0.4$

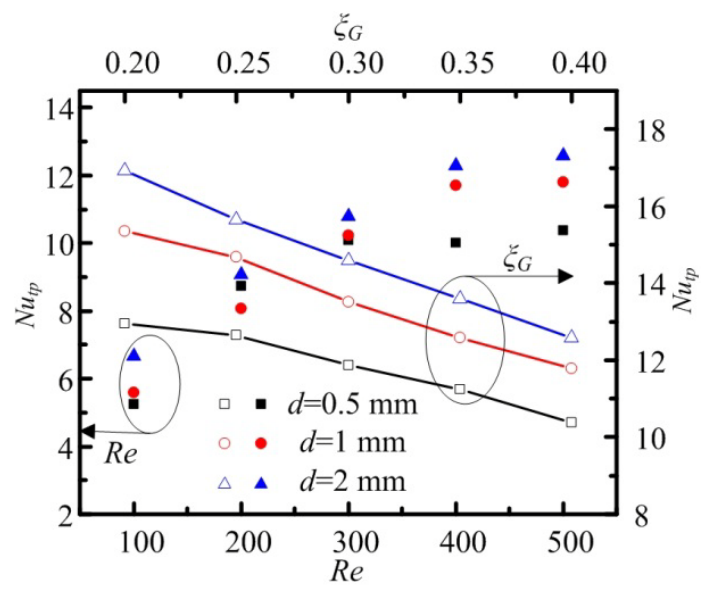

(a)

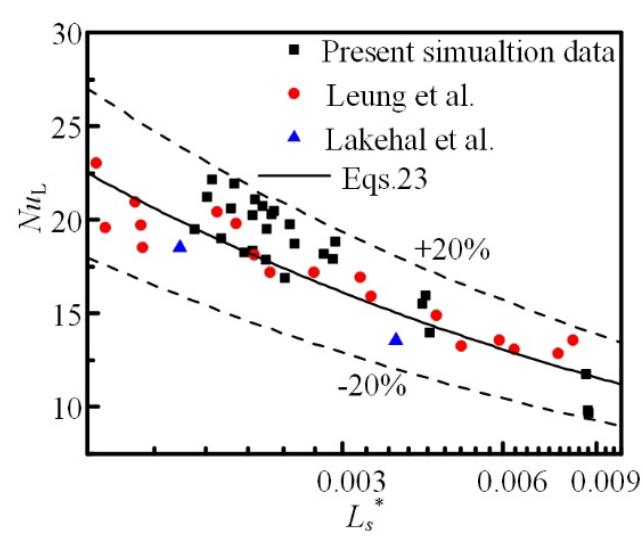

(b)

Fig. 10. (a) The effect of $R e$ and $\xi_{G}$ on $N u_{t p}$ (b) Comparison of average $N u_{l}{ }^{*}$ obtained from simulation and correlation. 